\title{
50 Jahre Schweizerische Gesellschaft für Anästhesiologie und Reanimation (SGAR)
}

T. Pasch, F. Frei

\section{Die Anästhesie nach 1945}

Unmittelbar nach dem 2. Weltkrieg wies die Anästhesie in fast allen kontinentaleuropäischen Ländern einen deutlichen Entwicklungsrückstand gegenüber den angloamerikanischen und skandinavischen Ländern auf. Die Medizin der Schweiz war schon vor dem Krieg durch Kontakte mit Deutschland, Frankreich und Österreich geprägt, die Fortschritte in der Anästhesiologie waren jedoch im angelsächsischen Sprachbereich erzielt worden. Das lag daran, dass im Krieg ein internationaler Austausch extrem erschwert, ja sogar unmöglich war und das Anästhesiewesen den Chirurgen oblag, die die Praxis ihren Narkoseschwestern anvertrauten. Gelegentlich mussten auch Assistenten Narkosen durchführen, wozu sich niemand drängte, weil die Narkose nicht als ärztliche Aufgabe angesehen wurde. Deshalb blieben Fortschritte aus, Lokal- und Leitungsanästhesien dominierten, und die Operationsindikationen mussten sehr eng gestellt werden.

Das Wissen davon, dass in England, wo die Durchführung der Narkose seit jeher dem Arzt vorbehalten war, in den USA und Kanada mit Hilfe von Curare und endotrachealer Intubation Allgemeinanästhesien durchgeführt würden, die den hier praktizierten Methoden haushoch überlegen seien, fand vorerst nur zögernd und punktuell Eingang in der Schweiz. Die Schweizerische Akademie der Medizinischen Wissenschaften unter dem Vorsitz von Prof. Gigon (Basel) sowie einzelne Chirurgen von Universitäten und Privatspitälern kamen schon in den vierziger Jahren zur Einsicht, dass im Narkosebereich ein grosser Nachholbedarf bestand. So fasste die SAMW 1946 den Beschluss, einen Schweizer Stipendiaten für längere Zeit nach Boston zu H. K. Beecher zu senden. W. Hügin, der sich bereits als chirurgischer Assistent mit Curare befasst hatte, erhielt das Stipendium und wurde nach anschliessenden Kurzaufenthalten in Schweden und England 1949 zum Leiter der Unterabteilung für Anästhesie an der Chirurgischen Universitätsklinik Basel ernannt. Einige junge Kollegen bildeten sich aus eigener Initiative aus, beispielsweise Ch. Bovay in den Vereinigten Staaten und K. Zeller in England. Ersterer liess sich in Lausanne in freier Praxis nieder, während Zeller 1949 die neu- geschaffene Stelle des leitenden Arztes für Anästhesie in Winterthur übernehmen konnte. K. Zimmermann lernte die moderne Narkose am Rotkreuzspital in Zürich von einem dort für ein Jahr tätigen englischen Anästhesisten kennen und bildete sich in diesem Fach zuerst als Mitarbeiter zweier englischer Kollegen und dann in England weiter. Danach war er 1951-75 Leiter der Anästhesie am Rotkreuzspital in Zürich, um 1976-1987 das Amt des Präsidenten des Zentralvorstandes der Verbindung der Schweizer Ärzte (FMH) auszuüben. F. Kern wurde 1951/52 in Kopenhagen und Oxford ausgebildet, übernahm danach die Anästhesieabteilung am Kantonsspital St. Gallen, wo er 1959 Chefarzt und erster Leiter einer selbständigen Anästhesieabteilung in der Schweiz wurde. Bern blieb, sowohl was die Universitätsklinik als auch die Privatspitäler anbelangt, während Jahren ein steiniger Boden. Gleiches galt - über lange Jahre - für die Universitätsklinik von Lausanne. Das Kantonsspital Genf engagierte 1949 für die chirurgische Klinik einen englischen Fachkollegen, der dort ein Jahr tätig war. Am Kantonsspital Zürich verlief die Entwicklung der modernen Anästhesie in Etappen. Hier führte schon seit 1937 der Neurochirurg H. Krayenbühl Intubationsnarkosen durch; er hatte diese Methode - quasi als Zugabe zu den operativen Techniken - in London erlernt. In der Chirurgie wendete K. Mülly die während eines thoraxchirurgischen Fortbildungshalbjahres in Stockholm erlernten Anästhesieverfahren für Thorax- und grosse Oberbauchoperationen erstmals im Herbst 1947 an. Er blieb aber Thoraxchirurg und zog sich wieder aus dem Anästhesiedienst zurück, als diesen G. Hossli ab 1951/52 zunehmend übernahm.

\section{Die Gründung der Fachgesellschaft}

Die wenigen Anästhesisten dieser Zeit wurden von den chirurgisch tätigen Kollegen recht unterschiedlich eingeschätzt. Einige waren von der Qualität der modernen Narkosen überzeugt, doch die Mehrzahl der Kollegen hatte kein Verständnis für die Entwicklung. Man war zufrieden mit dem Bestehenden, fürchtete um die etablierten Strukturen im Operationsbetrieb und an den 


\section{Abbildung 1}

Dr. Charles Bovay (geb. 1920), erster Präsident der SGAR.

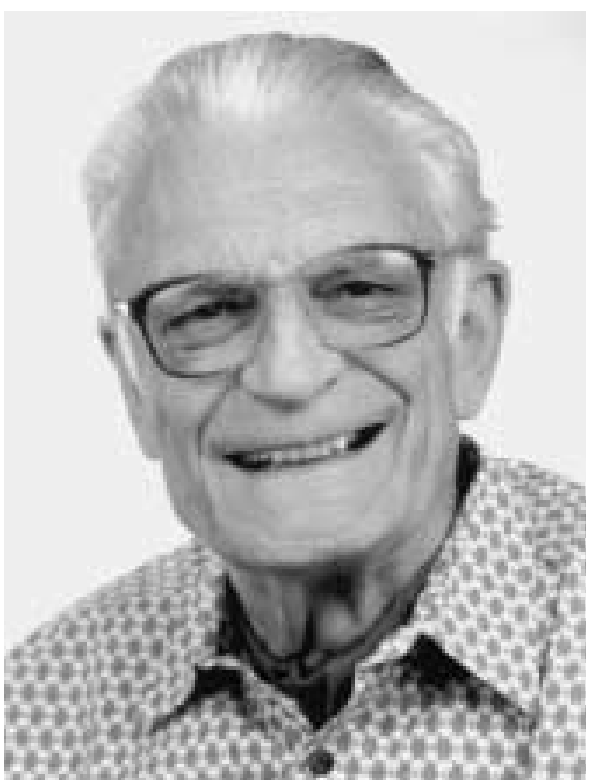

\section{Abbildung 2}

Dr. Karl Zimmermann (1922-1999), erster Aktuar der SGAR und 1976-1987 Präsident der FMH.

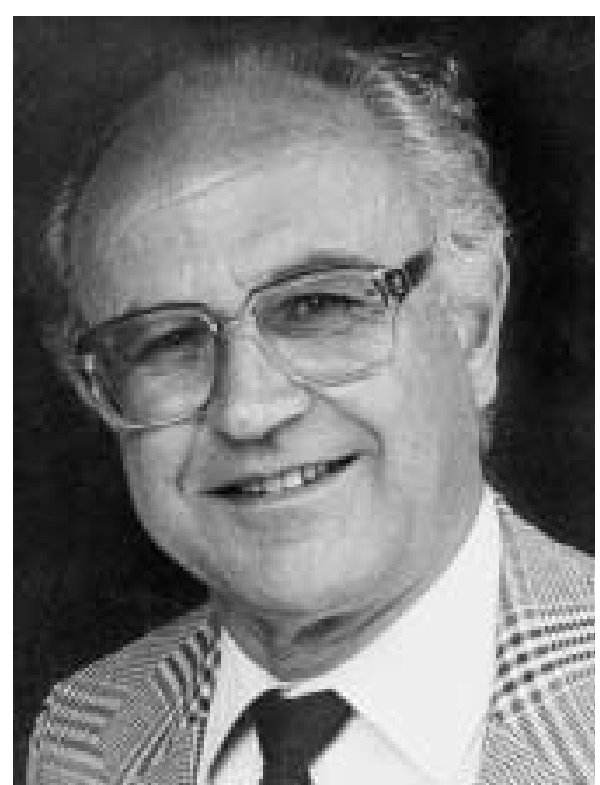

\section{Abbildung 3}

Prof. Werner Hügin (1918-2001), erster Kassier der SGAR.

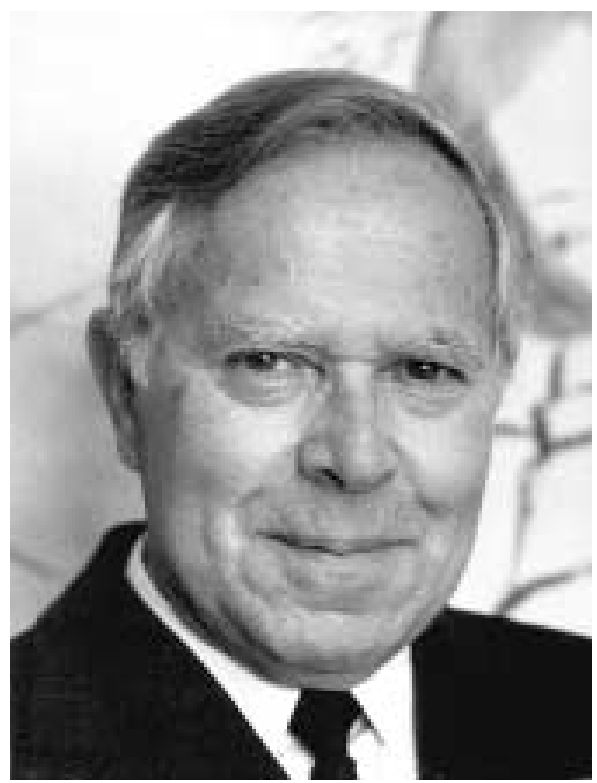

Kliniken, und nicht zuletzt gab es finanzielle Bedenken. All das veranlasste die kleine Anästhesistenschar zusammenzukommen, um sich gegenseitig kennenzulernen und die legitimen Interessen der neuen Spezialität gemeinsam zu vertreten. Am 11. Februar 1951 beschlossen Bovay, Hügin und Zeller in Bern, eine Berufsgemeinschaft schweizerischer Anästhesiologen (BSA) zu gründen. Hügin wurde mit der Ausarbeitung von Statuten betraut; diese wurden am 15. März 1951 angenommen und damit in Kraft gesetzt. Anlässlich der Jahrestagung der Schweizerischen Gesellschaft für Chirurgie vom 6./7. Juli 1951 hielt die BSA in Frauenfeld eine erste Generalversammlung $a b$, bei welcher weitere ordentliche und ausserordentliche Mitglieder aufgenommen wurden. Unter den ersteren waren P. König (Genf) und K. Zimmermann.

Schon in Frauenfeld wurde klar, dass die BSA wahrscheinlich nur kurzlebig sein würde, da die Schweizerische Gesellschaft für Chirurgie gleichentags eine Sektion für Anästhesiologie gegründet hatte, die sich vorwiegend mit den wissenschaftlichen Problemen der Anästhesiologie befassen sollte, während die beruflichen Fragen der BSA überlassen bleiben könnten. Doch die so bekundete Absicht der Chirurgen zum Dominieren erregte Widerspruchsgeist. Hügin übernahm es deshalb Anfang 1952, vorsorglicherweise einen Statutenentwurf für eine zu gründende Schweizerische Gesellschaft für Anästhesiologie mit Weiterbildungsvorschriften auszuarbeiten.
Dass am 19. Oktober 1951 eine Österreichische Gesellschaft für Anästhesiologie, ebenfalls gegen den Widerstand der Chirurgen, gegründet wurde, gab den Bestrebungen der Berufsgemeinschaft, sich in eine Gesellschaft umzuwandeln, zusätzlichen Auftrieb.

Im Juli 1952 fand in Zürich die 39. Jahresversammlung der Schweizerischen Gesellschaft für Chirurgie statt, zu welcher sie gemeinsam mit der BSA eingeladen hatte. Erstere hatte zusammen mit den Einladungsschreiben Statuten einer Sektion für Anästhesiologie verschickt, nach denen Anästhesisten dieser als zugewandte Mitglieder der Schweizerischen Gesellschaft für Chirurgie hätten beitreten können. Diese Tatsache und weitere Gründe führten dann gut ein Jahr nach Entstehen der BSA zur definitiven Gründung der Schweizerischen Gesellschaft für Anästhesiologie. Den Vorsitz der am 5. Juli 1952 stattfindenden Gründungsversammlung führte der Sekretär der BSA, K. Zeller. Die anwesenden ordentlichen Mitglieder stimmten dem Gründungsbeschluss und der Auflösung der BSA einhellig zu. Zum ersten Präsidenten wurde Ch. Bovay (Abb. 1), zum Aktuar K. Zimmermann (Abb. 2) und zum Kassier W. Hügin (Abb. 3) gewählt. Die vorgelegten Statuten wurden mit einer geringen Änderung angenommen und durch eine Urabstimmung im September 1952 definitiv in Kraft gesetzt. Die Gesellschaft umfasste damit sieben ordentliche, neun ausserordentliche und zwei zugewandte Mitglieder. 
Tabelle 1

Die Besetzung der Lehrstühle für Anästhesiologie an den Landesuniversitäten.

\begin{tabular}{|l|ll|ll|}
\hline Universität & Extraordinariat & \multicolumn{2}{l|}{ Ordinariat } \\
\hline Basel & 1963 & W. Hügin & 1965 & W. Hügin \\
& & & 1980 & M.B. Laver \\
& & & 1983 & D. Thomson \\
& & & 1989 & D. Scheidegger \\
\hline Bern & 1970 & B. Tschirren & 1972 & B. Tschirren \\
& & & 1988 & D. Thomson \\
& 1970 & M. Gemperle & 2003 & A. Kurz \\
\hline Genf & & & 1973 & M. Gemperle \\
& 1972 & J. Freeman & 1996 & F. Clergue \\
\hline Lausanne & & & 1978 & J. Freeman \\
& & & 2001 & D. R. Spahn \\
\hline Zürich & 1966 & G. Hossli & 1971 & G. Hossli \\
& & & 1987 & T. Pasch \\
\hline
\end{tabular}

\section{Frühe Entwicklungen}

Eine der ersten Aufgaben der neuen Fachgesellschaft war es, die Voraussetzungen für die Anerkennung als eigene Fachdisziplin zu schaffen. Sie erreichte, dass das erste offizielle Weiterbildungsprogramm für einen Spezialarzttitel FMH am 1. Januar 1954 in Kraft treten und bisherige Übergangsbestimmungen ersetzen konnte. Gefordert waren vier Jahre Weiterbildung, davon zwei Jahre in Anästhesiologie mit einem Auslandsobligatorium von sechs Monaten, das erst 1962 aufgehoben wurde. Weitere berufspolitische Probleme, die wie die Ausgestaltung der Weiterbildung die Gesellschaft in den folgenden Jahrzehnten immer wieder beschäftigen sollten, waren die Beziehungen zu den Chirurgen und anderen operativen Fächern, die Positionierung der Anästhesisten in der Spitalhierarchie und, damit eng verknüpft, die Anstellungs- und Vertragsbedingungen, Honorar- und Tariffragen, die $\mathrm{Zu}-$ sammenarbeit mit dem Anästhesiepflegepersonal, die Erweiterung der Tätigkeitsfelder auf Notfallfallmedizin, Intensivmedizin und Schmerztherapie und schliesslich auch die Entwicklung von Forschung und Lehre. In allen diesen Bereichen musste Kärrnerarbeit geleistet werden, die Fortschritte waren markant, aber auch Misserfolge blieben nicht aus. Als Bezeichnung des Fachgebietes setzte sich zunehmend der Begriff Anästhesiologie durch, mit dem die nicht auf die Narkose (Anästhesie im engeren Sinne) begrenzten, sondern auch die Intensiv- und Notfallmedizin und später auf die Schmerztherapie ein- beziehenden Aktivitäten subsumiert wurden, ohne dass auf diese neuen Felder ein Ausschliesslichkeitsanspruch erhoben wurde. 1967 erfolgte eine Namenserweiterung in Schweizerische Gesellschaft für Anästhesiologie und Reanimation (SGAR). In den sechziger Jahren kam es zur zunehmenden Verselbständigung durch organisatorische «Abnabelung» von der Chirurgie, allerdings nicht überall in gleichem Masse. 1967 erliess die SGAR «Richtlinien zum Vertragsabschluss zwischen Anästhesisten und Spitälern», in denen die Gleichstellung des Anästhesisten mit den anderen leitenden Ärzten im Spital gefordert wurde. Dieses Ziel wurde in den folgenden 35 Jahren aber nur langsam und nicht flächendeckend erreicht, weil manchenorts die Widerstände der Spitalleitungen und chirurgischen Chefärzte nicht überwunden werden konnten.

\section{Universitäre Einbindung}

Es dauerte 10-15 Jahre, bis sich die Anästhesiologie auch als akademisches Fach an den Landesuniversitäten voll etabliert hatte. 1957 konnten sich W. Hügin in Basel und M. Junod in Genf, 1960 G. Hossli in Zürich habilitieren. Lehrstühle wurden an den Schweizer medizinischen Fakultäten einige Jahre später eingerichtet. Als erster wurde Hügin 1963 zum Extraordinarius an der Basler Medizinischen Fakultät und Direktor des Instituts für Anästhesiologie des Kantonsspitals gewählt und zwei Jahre darauf zum Ordinarius befördert. Die weiteren Lehrstuhlinhaber für Anästhesiologie an den fünf medizinischen Fakultäten der Schweizer Universitäten sind in Tabelle 1 aufgeführt. Schon früh gab es in den deutschsprachigen Ländern gemeinsame Anstrengungen, durch wissenschaftliche Aktivität Anerkennung an der eigenen Hochschule sowie in nationalem und internationalem Rahmen zu finden. 1952 wurde die Zeitschrift «Der Anaesthesist» von R. Frey (Mainz), W. Hügin und O. Mayrhofer (Wien) begründet, zunächst als Organ der österreichischen, ein Jahr später auch der deutschen und der schweizerischen Fachgesellschaft. Die Anästhesisten der Romandie schufen sich mit einem jährlichen Sonderheft der Zeitschrift «Médecine et Hygiène» ein eigenes Publikationsforum. 1953 wurde beschlossen, künftig in zweijährlichem Rhythmus gemeinsame Tagungen der deutschen, österreichischen und schweizerischen Anästhesiegesellschaften zu veranstalten, woraus sich eine Tradition entwickelte, die wegen der «Globalisierung» der Wissenschaft $1995 \mathrm{zu}$ Ende ging. 


\section{Die weitere Entwicklung}

In den 50 Jahren des Bestehens der SGAR haben nicht nur die fachlich-medizinischen Aufgaben im engeren Sinne eine ständige Erweiterung und Vertiefung erfahren, sondern auch die berufspolitischen Rahmenbedingungen waren weiter $\mathrm{zu}$ entwickeln. Nicht selten hat die SGAR hierbei Tendenzen, die andere Fachgesellschaften und die FMH später vollzogen haben, vorweggenommen. Exemplarisch seien einige Aspekte aus den Bereichen Weiterbildung, Sicherheit und Qualitätssicherung beschrieben. 1984 wurde von der Generalversammlung der SGAR beschlossen, einen Antrag auf Einrichtung eines obligatorischen Fachexamens an die FMH zu stellen, und ein neues Prüfungsreglement wurde genehmigt. 1986 gab die FMH diesem Antrag statt, und seitdem gibt es in der Anästhesiologie als einem der ersten Fachgebiete in der Schweiz eine obligatorische, aus schriftlichem und mündlichem Teil bestehende Prüfung. Aus Gründen der Qualitätssicherung und -verbesserung schloss sich die SGAR für den schriftlichen Teil im Jahre 1988 an das 1984 eingeführte Examen der European Academy of Anaesthesiology an, dessen Bestehen Voraussetzung für die Verleihung des European Diploma in Anaesthesiology and Intensive Care ist. Die Academy war im Jahre 1978 als Organisation der akademisch engagierten Anästhesiologen in Europa gegründet worden. Dieses bewährte Vorgehen wurde allerdings erst mit dem am 1.1.1993 in Kraft getretenen Weiterbildungsprogramm zum Spezialarzttitel FMH für Anästhesiologie endgültig von der FMH sanktioniert. Diese machte ab 1.1.1995 für alle Fachgebiete eine Facharztprüfung zur Pflicht, also neun Jahre nach der Anästhesiologie. Seit 2000 lässt die SGAR-Kommission für die Anerkennung der Weiterbildungsstätten diese durch ein Visitationsteam alle sieben Jahre oder bei Wechsel des Leiters oder der Kategorie beurteilen. Auch dieser Initiative ist die FMH mit der Weiterbildungsordnung vom 1.1.2002 nachgefolgt.

Mitte 1986 verabschiedete der Vorstand der SGAR «Empfehlungen für die Personalbedarfsermittlung in der Anästhesie an öffentlichen Spitälern» sowie «Richtlinien für minimales apparatives Monitoring», wenige Monate vor den «Standards for Basic Intra-operative Monitoring» der American Society of Anesthesiologists, die bald weltweit Vorbildcharakter gewannen. Nach ausführlichen Diskussionen wurden 1993 «Standards und Empfehlungen» verabschiedet, in denen wichtige Fragen der Prozess- und Strukturqualität geregelt wurden, so beispielsweise die Personalstruktur, personelle Voraussetzungen und minimale Sicherheitsstandards. Diese neuen Leitlinien trugen durch ihren umfassenden Ansatz und logischen Aufbau wesentlich zur Beilegung von Missverständnissen und Kontroversen bei, welche 1990 verabschiedete «Grundsätze zur Kompetenzabgrenzung Anästhesiearzt/Anästhesiepflegepersonal» zwischen beiden Berufsgruppen hervorgerufen hatten, obwohl sie inhaltlich im Einklang mit dem Berufsbild Anästhesieschwester/Anästhesiepfleger des Schweizerischen Berufsverbandes der Krankenschwestern und Krankenpfleger (SBK) standen, das dessen Zentralvorstand und die SGAR 1989 genehmigt hatten. Im November 2002 wurden eine überarbeitete und erweiterte Fassung der Standards sowie Empfehlungen zur «Überwachung und Pflege nach Anästhesien» verabschiedet.

Ab 1995 wurde die Förderung der Qualität und Sicherheit der Anästhesie mit mehreren Projekten zielstrebig an die Hand genommen. Es wurde eine Kommission für Qualitätssicherung und -förderung gebildet. Diese entwickelte ein System zur gesamtschweizerischen Erfassung von Anästhesiedaten mittels eines «Minimal Data Set» sowie ein Meldesystem für kritische Anästhesieereignisse. Letzteres konnte 1998 als «Critical Incident Reporting System» (CIRS) auf der Website der SGAR installiert werden. Auch diese Anregung hat die FMH aufgegriffen und will ein allgemeines CIRS schaffen. Ein weiterer Schritt war 1999 die Schaffung einer Kommission zur Analyse von Anästhesie-Haftpflichtfällen. Sie sammelt und beurteilt abgeschlossene Haftpflichtfälle nach einem fixen Schema und übermittelt diese Informationen den praktisch tätigen Anästhesieärzten als Beitrag zur Verbesserung der Patientensicherheit. Aufgrund des Einsatzes des Kommissionspräsidenten H. Schaer erklärten sich die grossen Haftpflichtversicherer zur Mitarbeit bereit. Schliesslich wurde auf Initiative von B. Meister im Januar 2001 eine «Stiftung Patientensicherheit in der Anästhesie» gegründet, zu deren Stiftungsratspräsident er gewählt wurde. Stiftungszweck ist die Unterstützung von Aktivitäten, die geeignet sind, die Sicherheit von Anästhesien für Patienten zu erhöhen; insbesondere sollen die Erkenntnisse aus diesen Aktivitäten allen interessierten Kreisen zugänglich gemacht werden. Als Träger der Stiftung konnten ausser der SGAR die Schweizerische Patienten-Organisation, der Schweizerische Versicherungsverband sowie pharmazeutische und medizintechnische Firmen gewonnen werden. In den Print- und elektronischen Medien fand die Lancierung dieser Stiftung einen erfreulichen Widerhall; ihr wurde Beispielcharakter zuerkannt. 


\section{Gegenwart und Zukunft}

Aus heutiger Sicht ist den 14 Ärzten, die 1952 die Schweizerische Gesellschaft für Anästhesiologie gegründet haben, Mut und Weitsicht zu bescheinigen. Sie haben eine Entwicklung in Gang gesetzt, die sie erhoffen, aber zum damaligen Zeitpunkt nicht erwarten durften. Aus einem unscheinbaren Samenkorn ist in den seither vergangenen 50 Jahren ein lebenskräftiger Baum mit solidem Stamm und vielen Ästen, Blüten und Früchten geworden. Aus der Schweizerischen Gesellschaft für Anästhesiologie des Jahres 1952 ist die Schweizerische Gesellschaft für Anästhesiologie und Reanimation des Jahres 2002 mit über 700 Mitgliedern entstanden. Sie ist in 50 Jahren auf das 50fache gewachsen, wie die Tabelle 2 zeigt.

Im Jahre 2002 präsentiert sich die schweizerische Anästhesiologie als ein eigenständiges, selbstbewusstes und attraktives Spezialgebiet der Medizin. Sie ist nicht auf die Ausführung von Anästhesien im engeren Sinne beschränkt, sondern wesentlich an der prä- und postoperativen Betreuung von Patienten beteiligt, das Berufsbild hat sich in Richtung perioperative Medizin entwickelt. Intensiv- und Notfallmedizin sowie die Behandlung akuter und chronischer Schmerzen sind etablierte Komponenten des Fachgebiets, ohne dass auf diese Felder ein Ausschliesslichkeitsanspruch erhoben wird. Anästhesiologen haben sich innerhalb der "Medical Community» Anerkennung erworben und an Gewicht gewonnen; früher fast alltägliche, die Selbst- und Fremdeinschätzung gefährdende Kontroversen mit operativen Fachgebieten bzw. deren Vertretern sind seltener und sachlicher geworden; eine wechselseitige Wertschätzung mit Anerkennung der Fachkompetenz und der Verantwortlichkeiten des Partners ist die Regel und nicht die Ausnahme. Nicht nur aus nationalem, sondern auch aus internationalem Blickwinkel ist die wissenschaftliche Reputation gut, es gelten effektive Sicherheitsstandards, Weiter- und Fortbildung zeichnen sich durch hohe Qualität aus.

Mehr und mehr wird der schnelle Wandel ein bestimmender Faktor der Tätigkeit. Dieser betrifft die klinischen Verfahren und die wissenschaftlichen Grundlagen sowohl des Fachgebietes selbst als auch der Nachbar- und operativen Partnerfächer und schliesslich die Medizin insgesamt. Nur zu einem Teil sind die Fortschritte der Medizin für die Geschwindigkeit des Wandels verantwortlich, vielmehr ist das Gesamt-
Tabelle 2

Dekadische Entwicklung der Mitgliederzahlen (alle Kategorien) der SGAR.

\begin{tabular}{|l|l|}
\hline Jahr & Mitgliederzahl \\
\hline 1952 & 14 \\
1962 & 77 \\
1972 & 154 \\
1982 & 296 \\
1992 & 478 \\
2002 & 715 \\
\hline
\end{tabular}

gefüge des Gesundheitswesens in einem ständigen Umbruch begriffen, der von unterschiedlichen gesellschaftlichen, wirtschaftlichen und politischen Kräften getrieben wird, ohne eine klare Richtung zu haben. Mehr und mehr wird das nicht nur von Anästhesiologen als Fremdbestimmung wahrgenommen, der viele sich schicksalhaft ausgeliefert sehen. Als aktuelle drängende Probleme sind zu nennen: Änderungen des Tarif- und Vergütungssystems; zunehmende Diskrepanz zwischen Spital- oder Abteilungsbudgets und ständig wachsenden Leistungsansprüchen; legale Zwänge; dauernd zunehmender formalistischer Dokumentationsaufwand; Arbeitszeitreglementierungen und ihre Auswirkungen auf die Arbeits- und Weiterbildungsqualität; Schwierigkeiten der Nachwuchsrekrutierung einerseits und Furcht vor einem Überangebot an Fachärzten andererseits - wobei in vielen europäischen Ländern ein bedrohlicher Mangel an Anästhesiologen prognostiziert wird.

Diesen Herausforderungen muss und will sich die SGAR stellen, indem sie ihre bisherigen Stärken, nämlich Entwicklungen früh zu erkennen und mitzugestalten, Ziele langfristig zu planen und umzusetzen, erhält und weiter ausbaut. Die bereits erwähnte Stiftung für Patientensicherheit in der Anästhesie ist ein Paradebeispiel für ein in die Zukunft weisendes Projekt, dessen Pioniercharakter daran erkennbar ist, dass die FMH zusammen mit Bundesbehörden ein vergleichbares Projekt auf nationaler Ebene inaugurieren will. Am 14. November 2002 hat die SGAR bei ihrem Jahreskongress in Lausanne ihr 50jähriges Bestehen mit einem Festakt gefeiert und zu diesem Anlass eine Festschrift herausgegeben, deren Autoren nicht nur Rückschau auf die historische Entwicklung halten, sondern auch eine Standortbestimmung des Faches und der Fachgesellschaft für das Jahr 2002 vornehmen [1].
Frei F, Pasch 1, Spahn DR Hans Huber, Bern Göttingen, Toronto, Seattle 2002 